\title{
ESTABILIZACIÓN DEL MOVIMIENTO DE UN CUERPO VISCOELÁSTICO BAJO UN CONTROL
}

\author{
Yolanda Santiago*
}

\begin{abstract}
Resumen: En este trabajo probamos la estabilidad exponencial del movimiento de un cuerpo viscoelástico bajo un control, usando la teoría de estabilización de ecuaciones integrodiferenciales introducidas por Desh [2].

Palabras clave: Ecuaciones integrodiferenciales, sistemas viscoelásticos, estabilización exponencial, crecimiento esencial del resolvente.
\end{abstract}

\section{STABILIZATION OF THE MOTION OF A VISCOELASTIC BODY UNDER}

\begin{abstract}
In this article, we prove the exponential stability of the motion of a viscoelastic body under control, using the stabilization theory of integro differential equations, introduced by Desh [2].
\end{abstract}

Key words: Integro differential equations, viscoelastic system, exponential stabilization, essential growth of the resolvent.

\section{Introducción}

En nuestro marco teórico estudiamos la existencia de solución y la estabilización de la ecuación integrodiferencial

$$
u_{t t}=-\alpha_{1} A u(t)+\alpha_{2} k * A u+f(t)
$$

al cual se le suministra un control $f(t)=-B_{0} u(t)-B_{1} u_{t}(t)$, donde los $B_{i}$ son operadores acotados lineales y de rango finito, $A$ es un operador no acotado autoadjunto positivo y $k$ el núcleo de relajación que representa la memoria del material. Aquí estamos representando al operador $*$ como la convolución en el tiempo. Se hace uso del concepto tasa de crecimiento esencial para operadores resolventes que viene a ser una extensión de lo referente a semigrupos de clase $C_{o}$, citamos a Desh [2]. Focalizamos entonces nuestro estudio al caso

$$
u_{t t}=-\alpha_{1} A u(t)-\frac{\alpha_{2}}{\alpha_{1}} k(0) u_{t}(t)+f(t)
$$

y estudiamos su crecimiento esencial el cual reflejará el crecimiento esencial del operador resolvente de (1). En seguida estudiamos la estabilización del movimiento de un cuerpo viscoelástico bajo efectos de un control.

Inspirados en [1], nuestra inclinación y motivación a este problema mecánico nos lleva a considerar un control óptimo en la búsqueda de obtener estabilización exponencial. Se sabe que para el caso elástico, la estabilización exponencial es imposible, ver [17 ]. Por otro lado, la ecuación (2) puede ser observada como una ecuación de la onda y físicamente representa a un sólido viscoelástico tipo Kelvin-Voigt, esto motiva la posibilidad de dar una respuesta afirmativa con respecto a la estabilización exponencial de la ecuación (1), citamos [3]. El concepto de "tasa de crecimiento esencial" se puede encontrar en [7], [9 ], [ 16], [8 ] y [19 ]. Para el estudio de las ecuaciones de Volterra en espacios abstractos usamos [4], [5] y [6]. Finalmente, también podemos citar los trabajos donde se aborda el comportamiento asintótico de algunos sistemas de evolución: [10], [11], [12], [13], [14] y [15].

\footnotetext{
${ }^{*}$ UNMSMS, Facultad de Ciencias Matemáticas, e-mail: ysantiagoa@unmsm.edu.pe
} 


\section{Marco Teórico: Ecuación Integrodiferencial}

Sea $X$ un espacio de Hilbert, consideramos la ecuación integrodiferencial de Volterra de segundo orden:

$$
\begin{aligned}
U^{\prime \prime}(t) & =-\alpha_{1} A U(t)+\alpha_{2} \int_{0}^{t} k(t-s) A U(s) d s+f(t) \\
U(0) & =u_{0} \\
U_{t}(0) & =u_{1}
\end{aligned}
$$

donde $A$ es un operador densamente definido, semidefinido positivo y autoadjunto, de modo que para algún $\lambda \in \mathbb{R}$, el operador $\left(\lambda^{2} I-A\right)^{-1}$ sea compacto.

En particular, $A$ tiene espectro discreto consistente de autovalores $0 \leq \lambda_{1}^{2} \leq \lambda_{2}^{2} \leq \ldots$ con $0 \leq \lambda_{i} \rightarrow+\infty$ cuando $i \rightarrow+\infty$ y denotamos por $e_{i}$ los correspondientes autovectores.

Consideramos $0<\alpha_{1}<\alpha_{2}$ escalares y $k:[0, L] \rightarrow[0, \infty)$ una función tal que para algún $\widetilde{k}>0$ la función $k(t)$ sea positiva y decreciente, satisfaciendo:

i) $\int_{0}^{\infty} k(t) d t=1$

ii) $\int_{0}^{\infty} e^{\widetilde{k} t}\left|k^{\prime}(t)\right| d t<\infty$

iii) $\alpha_{2} \widehat{k}(\lambda) \neq \alpha_{1}$ para $\operatorname{Re} \lambda \geq-\widetilde{k}$

iv) $k(0)<\infty$.

Además, consideramos $k^{\prime}$ de variación acotada o $k$ convexa.

Asumimos: $u_{0} \in \operatorname{dom} A^{\frac{1}{2}}$ y $u_{1} \in X$.

Bajo estas consideraciones tratamos de dar respuesta al siguiente problema.

Problema

¿Es posible estabilizar exponencialmente el sistema (??) considerando

$$
f(t)=-C_{0} U(t)-C_{1} U_{t}(t) ?
$$

donde para cada $i=1,2, \operatorname{los} C_{i}$ son operadores lineales compactos, autoadjuntos y semidefinidos positivos en $X$.

La respuesta a este problema es afirmativa.

Para ello, escribiremos (??) como un sistema de primer orden.

Consideramos

$$
\mathcal{X}:=\operatorname{dom} A^{\frac{1}{2}} \times X
$$

y

$$
V(t):=\left(\begin{array}{c}
U(t) \\
U_{t}(t)
\end{array}\right)
$$

Luego,

$$
\begin{aligned}
V_{t}(t)= & \left(\begin{array}{c}
U_{t}(t) \\
U_{t t}(t)
\end{array}\right) \\
= & \left(\begin{array}{c}
U_{t}(t) \\
-\alpha_{1} A U(t)+\alpha_{2} \int_{0}^{t} k(t-s) A U(s) d s+-C_{0} U(t)-C_{1} U_{t}(t)
\end{array}\right) \\
= & \underbrace{\left(\begin{array}{cc}
0 & I \\
-\alpha_{1} A & 0
\end{array}\right)}_{\mathcal{A}:=}\left(\begin{array}{c}
U(t) \\
U_{t}(t)
\end{array}\right) \\
& +\int_{0}^{t} F(t-s) \mathcal{A} V(s) d s+\underbrace{\left(\begin{array}{cc}
0 & 0 \\
-C_{0} & -C_{1}
\end{array}\right)}_{C:=}\left(\begin{array}{c}
U(t) \\
U_{t}(t)
\end{array}\right)
\end{aligned}
$$


donde

$$
\operatorname{dom} \mathcal{A}:=\operatorname{dom} A \times \operatorname{dom} A^{\frac{1}{2}}
$$

y

$$
F(t):=\left(\begin{array}{cc}
0 & 0 \\
0 & -k(t) \frac{\alpha_{2}}{\alpha_{1}}
\end{array}\right)
$$

Así, el sistema de primer orden quedaria expresada por

$$
\begin{aligned}
& V^{\prime}(t)=\mathcal{A} V(t)+\int_{0}^{t} F(t-s) A V(s) d s+C V(t) \\
& V(0)=\left(\begin{array}{c}
U(0) \\
U_{t}(0)
\end{array}\right)=\left(\begin{array}{l}
u_{0} \\
u_{1}
\end{array}\right)
\end{aligned}
$$

Entonces el problema (??) esta bien puesto y además se cumple el siguiente resultado:

Teorema 2.1. Suponiendo que $C_{0}$ y $C_{1}$ satisfacen:

1. $\operatorname{Ker} C_{0} \cap \operatorname{Ker} A=\{0\}$

2. El subespacio $\operatorname{Ker} C_{1} \cap \operatorname{Ker} A$ no contiene autovectores de $C_{0}$

Entonces (??) es exponencialmente estable, i.e. $U$ y $U_{t}$ son exponencialmente estables.

\section{Estabilización exponencial del movimiento de un cuerpo viscoelástico}

Consideraremos un cuerpo rígido con masa $M$ y anexado a él una vara viscoelástica axialmente flexible (i.e. un apéndice).

Consideraremos la siguiente ecuación constitutiva viscoelástica lineal para la tensión en el apéndice,

$$
\sigma(t, z)=\alpha_{1} \frac{\partial y}{\partial z}(t, z)-\alpha_{2} \int_{0}^{t} k(t-s) \frac{\partial y}{\partial z}(s, z) d s
$$

donde $\alpha_{1}, \alpha_{2}$, y $k$ satisfacen las propiedades de la sección anterior.

El control es suministrado por una fuerza $f(t)$ actuando en el cuerpo rígido.

Obtenemos el siguiente sistema de ecuaciones:

$$
\begin{aligned}
& (M+\rho L) \frac{\partial^{2} x}{\partial t^{2}}(t)+\rho \int_{0}^{L} \frac{\partial^{2} y}{\partial t^{2}}(t, z) d z=f(t) \\
& \rho\left(\frac{\partial^{2} y}{\partial t^{2}}(t, z)+\frac{\partial^{2} x}{\partial t^{2}}(t)\right)=\alpha_{1} a \frac{\partial^{2} y}{\partial z^{2}}(t, z)-\alpha_{2} a \int_{0}^{t} k(t-s) \frac{\partial^{2} y}{\partial z^{2}}(s, z) d s \\
& y(t, 0)=0, \frac{\partial y}{\partial z}(t, L)=0
\end{aligned}
$$

y consideraremos un control negativo dependiente de la posición y velocidad del cuerpo rígido:

$$
f(t)=-c_{o} x(t)-c_{1} \frac{\partial x}{\partial t}(t)
$$


donde $c_{0}>0$ y $c_{1} \geq 0$.

Por otro lado, integrando respecto a $z$, desde 0 a $L$, la ecuación (??) y usando Fubini obtenemos

$$
\begin{aligned}
& \rho \int_{0}^{L} \frac{\partial^{2} y}{\partial t^{2}}(t, z) d z+\rho \frac{\partial^{2} x}{\partial t^{2}}(t) L \\
& \quad=\alpha_{1} a \int_{0}^{L} \frac{\partial^{2} y}{\partial z^{2}}(t, z) d z-\alpha_{2} a \int_{0}^{L} \int_{0}^{t} k(t-s) \frac{\partial^{2} y}{\partial z^{2}}(s, z) d s d z \\
& \quad=\left.\alpha_{1} a \frac{\partial y}{\partial z}(t, z)\right|_{0} ^{L}-\alpha_{2} a \int_{0}^{t} k(t-s)\left(\int_{0}^{L} \frac{\partial^{2} y}{\partial z^{2}}(s, z) d z\right) d s \\
& \quad=\alpha_{1} a(\underbrace{\frac{\partial y}{\partial z}(t, L)}_{=0}-\frac{\partial y}{\partial z}(t, 0))-\alpha_{2} a \int_{0}^{t} k(t-s)\left(\left.\frac{\partial y}{\partial z}(s, z)\right|_{0} ^{L}\right) d s \\
& \quad=-\alpha_{1} a \frac{\partial y}{\partial z}(t, 0)-\alpha_{2} a \int_{0}^{t} k(t-s) \underbrace{\frac{\partial y}{\partial z}(s, L)}_{=0}-\frac{\partial y}{\partial z}(s, 0)) d s \\
& \quad=-\alpha_{1} a \frac{\partial y}{\partial z}(t, 0)+\alpha_{2} a \int_{0}^{t} k(t-s) \frac{\partial y}{\partial z}(s, 0) d s .
\end{aligned}
$$

De la ecuación (??) y usando la igualdad (??) obtenemos

$$
M \frac{\partial^{2} x}{\partial t^{2}}(t)=f(t)+\alpha_{1} a \frac{\partial y}{\partial z}(t, 0)-\alpha_{2} a \int_{0}^{t} k(t-s) \frac{\partial y}{\partial z}(s, 0) d s .
$$

Introducimos

$$
w(t, z):=x(t)+y(t, z)
$$

entonces

$$
\frac{\partial w}{\partial z}(t, z)=\frac{\partial y}{\partial z}(t, z)
$$

en particular:

$$
\begin{aligned}
\frac{\partial w}{\partial z}(t, 0) & =\frac{\partial y}{\partial z}(t, 0) \\
\frac{\partial w}{\partial z}(t, L) & =\frac{\partial y}{\partial z}(t, L) .
\end{aligned}
$$

Usando (??) tenemos

$$
\frac{\partial^{2} x}{\partial t^{2}}(t)=M^{-1} f(t)+M^{-1} \alpha_{1} a \frac{\partial w}{\partial z}(t, 0)-M^{-1} \alpha_{2} a \int_{0}^{t} k(t-s) \frac{\partial w}{\partial z}(s, 0) d s .
$$

Igualmente, como $w(t, z)=x(t)+y(t, z)$ tenemos que

$$
\begin{aligned}
\frac{\partial w}{\partial t}(t, z) & =\frac{\partial x}{\partial t}(t)+\frac{\partial y}{\partial t}(t, z) \\
\frac{\partial^{2} w}{\partial t^{2}}(t, z) & =\frac{\partial^{2} x}{\partial t^{2}}(t)+\frac{\partial^{2} y}{\partial t^{2}}(t, z) \\
\frac{\partial^{2} w}{\partial z^{2}}(t, z) & =\frac{\partial^{2} y}{\partial z^{2}}(t, z) .
\end{aligned}
$$

Usando (??) y (??) en (??), obtenemos

$$
\frac{\partial^{2} w}{\partial t^{2}}(t, z)=\rho^{-1} \alpha_{1} a \frac{\partial^{2} w}{\partial z^{2}}(t, z)-\rho^{-1} \alpha_{2} a \int_{0}^{t} k(t-s) \frac{\partial^{2} w}{\partial z^{2}}(s, z) .
$$




\section{ESTABILIZACIÓN EXPONENCIAL DEL MOVIMIENTO DE UN CUERPO VISCOELÁSTICO91}

Ahora afirmamos que

$$
\frac{\partial w}{\partial z}(t, L)=0, \quad w(t, 0)=x(t) .
$$

En efecto, de (??) y (??) tenemos que $\frac{\partial w}{\partial z}(t, L)=\frac{\partial y}{\partial z}(t, L)=0$. Por otro lado, $w(t, 0)=x(t)+\underbrace{y(t, 0)}_{=0}=$ $x(t)$.

Considerando

$$
U(t):=\left(\begin{array}{c}
x(t) \\
w(t, \cdot)
\end{array}\right)
$$

un vector en $X=\mathbb{R} \times L^{2}([0, L], \mathbb{R})$, escribimos el sistema (??), (??) y (??) como la ecuación de segundo orden:

$$
U_{t t}=-\alpha_{1} A U(t)+\alpha_{2} \int_{0}^{t} k(t-s) A U(s) d s-C_{0} U(t)-C_{1} U_{t}(t)
$$

donde $A$ es el operador definido por:

$$
\begin{gathered}
A U(t):=A\left(\begin{array}{c}
x(t) \\
w(t, \cdot)
\end{array}\right):=\left(\begin{array}{c}
-M^{-1} a \frac{\partial w}{\partial z}(t, 0) \\
-\rho^{-1} a \frac{\partial^{2} w}{\partial z^{2}}(t, z)
\end{array}\right), \\
\operatorname{dom} A:=\left\{(x, w)^{T} \in X, w \in W^{2,2}([0, L], \mathbb{R}), w(0)=x, \frac{\partial w}{\partial z}(L)=0\right\}
\end{gathered}
$$

y

$$
C_{i} U(t)=C_{i}\left(\begin{array}{c}
x(t) \\
w(t, \cdot)
\end{array}\right):=\left(\begin{array}{c}
M^{-1} c_{i} x(t) \\
0
\end{array}\right)
$$

para $i=0,1$.

En $X$ introducimos el producto interno

$$
\begin{aligned}
<U_{1}, U_{2}>: & =M x_{1} x_{2}+\rho \int_{0}^{L} w_{1}(z) w_{2}(z) d z \\
& =M<x_{1}, x_{2}>_{\mathbb{R}}+\rho<w_{1}, w_{2}>_{L^{2}([0, L], \mathbb{R})} .
\end{aligned}
$$

El espacio $X$ con este producto interno es un espacio de Hilbert.

Observamos que el sistema (??) encaja en el modelo (??). Con todo esto probaremos los siguientes resultados.

Proposición 3.1. El operador A es autoadjunto y semidefinido positivo $(<U, A U\rangle \geq 0, \forall U \in D(A))$. Prueba.

a) $A$ es autoadjunto. En efecto,

1. $A^{*} \subset A$

Para $Z_{2}=\left(x_{2}, w_{2}\right)^{T} \in \operatorname{dom} A^{*}, U=(u, v)^{T}=A^{*} Z_{2}$ y cualquier $Z_{1}=\left(x_{1}, w_{1}\right)^{T} \in \operatorname{dom} A$, tenemos:

$$
<U, Z_{1}>=<A^{*} Z_{2}, Z_{1}>=<Z_{2}, A Z_{1}>
$$

Entonces

$$
\begin{aligned}
M u x_{1} & +\rho \int_{0}^{L} v(z) w_{1}(z) d z \\
= & -x_{2} a \frac{\partial w_{1}}{\partial z}(t, 0)-a \int_{0}^{L} w_{2}(z) \frac{\partial^{2} w_{1}}{\partial z^{2}}(t, z) d z
\end{aligned}
$$


Tomaremos primero $w_{1} \in C_{0}^{\infty}([0, L], \mathbb{R})$ infinitamente diferenciable con soporte compacto en $[0, L]$ y $x_{1}=0$. Así, de (??) obtenemos:

$$
\begin{aligned}
\rho \int_{0}^{L} v(z) w_{1}(z) d z & =-a \int_{0}^{L} w_{2}(z) \frac{\partial^{2} w_{1}}{\partial z^{2}}(t, z) d z \\
& =a \int_{0}^{L} \frac{\partial w_{2}}{\partial z}(z) \frac{\partial w_{1}}{\partial z}(t, z) d z+0 \\
& =-a \int_{0}^{L} \frac{\partial^{2} w_{2}}{\partial z^{2}}(z) w_{1}(t, z) d z
\end{aligned}
$$

i.e.

$$
\int_{0}^{L}\left\{v(z)+a \rho^{-1} \frac{\partial^{2} w_{2}}{\partial z^{2}}(z)\right\} w_{1}(z) d z=0, w_{1} \in C_{0}^{\infty}([0, L], \mathbb{R})
$$

Así,

$$
v=-a \rho^{-1} w_{2}^{\prime \prime},
$$

siendo la derivada en el sentido distribucional.

Como $w_{2} \in L^{2}([0, L], \mathbb{R})$ y $w_{2}^{\prime \prime} \in L^{2}([0, L], \mathbb{R})$ por Du bois Raymond, entonces por derivadas intermediarias $w_{2} \in W^{2,2}([0, L], \mathbb{R})$.

Ahora, para cualquier $w_{1} \in W^{2,2}([0, L], \mathbb{R})$ con $w_{1}^{\prime}(L)=0$, tomamos $x_{1}:=w_{1}(0)$, tal que $\left(x_{1}, w_{1}\right)^{T} \in \operatorname{dom} A$, y luego hallando la integral en la igualdad (??), usando la expresion de $v$ de (??) e integrando por partes, obtenemos

$$
\begin{aligned}
M u & \underbrace{x_{1}}_{=w_{1}(0)}-a \int_{0}^{L} w_{2}^{\prime \prime}(z) w_{1}(z) d z \\
= & -a x_{2} w_{1}^{\prime}(0)-a w_{2}(L) \underbrace{w_{1}^{\prime}(L)}_{=0}+a w_{2}(0) w_{1}^{\prime}(0) \\
& +a w_{2}^{\prime}(L) w_{1}(L)-a w_{2}^{\prime}(0) w_{1}(0)-a \int_{0}^{L} w_{2}^{\prime \prime}(z) w_{1}(z) d z .
\end{aligned}
$$

Es decir,

$$
\begin{aligned}
& M u w_{1}(0)=-a x_{2} w_{1}^{\prime}(0)+a w_{2}(0) w_{1}^{\prime}(0) \\
& +a w_{2}^{\prime}(L) w_{1}(L)-a w_{2}^{\prime}(0) w_{1}(0),
\end{aligned}
$$

que factorizando nos da:

$$
\left[M u+a w_{2}^{\prime}(0)\right] w_{1}(0)+\left[x_{2}-w_{2}(0)\right] a w_{1}^{\prime}(0)-\left[w_{2}^{\prime}(L)\right] a w_{1}(L)=0
$$

Como (??) se verifica $\forall w_{1} \in W^{2,2}([0, L], \mathbb{R})$ con $w_{1}^{\prime}(L)=0$, entonces deducimos que

$$
\begin{aligned}
& x_{2}=w_{2}(0), \\
& w_{2}^{\prime}(L)=0, \\
& u=-M^{-1} a w_{2}^{\prime}(0) .
\end{aligned}
$$

Usando esto y (??) tenemos que $A^{*} Z_{2}=(u, v)^{T}=U=A Z_{2}$, y $Z_{2} \in \operatorname{dom} A$

2. $A \subset A^{*}$

Dado $Z_{1}=\left(x_{1}, w_{1}\right)^{T}, Z_{2}=\left(x_{2}, w_{2}\right)^{T} \in \operatorname{dom} A$, integrando por partes y como $x_{i}=w_{i}(0) \mathrm{y}$ $w_{i}^{\prime}(L)=0$ para $i=1,2$, tenemos

$$
\begin{aligned}
<Z_{1}, A Z_{2}>= & -a x_{1} w_{2}^{\prime}(0)-a \int_{0}^{L} w_{1}(z) w_{2}^{\prime \prime}(z) d z \\
= & -a w_{1}(0) w_{2}^{\prime}(0)+w_{1}(0) w_{2}^{\prime}(0) \\
& +a \int_{0}^{L} w_{1}^{\prime}(z) w_{2}^{\prime}(z) d z \\
= & -a w_{1}^{\prime}(0) x_{2}-a \int_{0}^{L} w_{1}^{\prime \prime}(z) w_{2}(z) d z \\
= & \left.<A Z_{1}, Z_{2}\right\rangle .
\end{aligned}
$$




\section{ESTABILIZACIÓN EXPONENCIAL DEL MOVIMIENTO DE UN CUERPO VISCOELÁSTICO93}

Entonces $Z_{2} \in \operatorname{dom} A^{*}$ y $A^{*} Z_{2}=A Z_{2}$.

De ambos items 1. y 2. tenemos que $A=A^{*}$.

b) $A$ es semidefinido positivo en $X$, i.e. $\langle U, A U>\geq 0, \forall U \in \operatorname{dom} A$. En efecto,

$$
<\left(\begin{array}{c}
x \\
w
\end{array}\right), A\left(\begin{array}{c}
x \\
w
\end{array}\right)>=M x(-1) M^{-1} a w^{\prime}(0)-a \underbrace{\int_{0}^{L} w(z) w^{\prime \prime}(z) d z}_{J:=} .
$$

Ahora, como $(x, w)^{T} \in \operatorname{dom} A$, tenemos que $w(0)=x$ y $w^{\prime}(L)=0$. Luego,

$$
\begin{aligned}
J & =-\int_{0}^{L} w^{\prime}(z) w^{\prime}(z) d z+\left.w(z) w^{\prime}(z)\right|_{0} ^{L} \\
& =-\int_{0}^{L} w^{\prime}(z) w^{\prime}(z) d z-x w^{\prime}(0) .
\end{aligned}
$$

Substituyendo $J$ en la identidad previa tenemos,

$$
\begin{aligned}
<\left(\begin{array}{c}
x \\
w
\end{array}\right), A\left(\begin{array}{l}
x \\
w
\end{array}\right)> & =a \int_{0}^{L} w^{\prime}(z) w^{\prime}(z) d z \\
& =a \int_{0}^{L}\left|w^{\prime}(z)\right|^{2} d z \geq 0 .
\end{aligned}
$$

Observación 3.1. Se verifican las siguientes propiedades

i) $\operatorname{dom} A^{\frac{1}{2}}$ es la completación de dom A bajo la norma de $W^{1,2}$,

$$
\operatorname{dom} A^{\frac{1}{2}}=\left\{(x, w)^{T} \in X, w \in W^{1,2}([0, L], \mathbb{R}) \text { y } x=w(0)\right\} .
$$

ii) $\forall \lambda>0,(\lambda I-A)^{-1}$ es compacto, desde que $W^{2,2}$ está inmerso compactamente en $L^{2}$.

Proposición 3.2. Para cada $i=1,2$, el operador $C_{i}$ es compacto, semidefida positivo y autoadjunto en $X$.

\section{Prueba.}

i) Para $i=1,2, C_{i}$ es un operador lineal de rango finito, luego es compacto. En efecto $\operatorname{dim} C_{i}(X)=1$

ii) $C_{i}$ es semidefinido positivo y autoadjunto. En efecto, $\left\langle U, C_{i} U\right\rangle=M x M^{-1} c_{i} x=c_{i} x^{2} \geq 0$, para $U=(x, w)^{T} \in X=\operatorname{dom} C_{i}$. Para probar que $C_{i}$ es autoadjunto, primero veremos fácilmente que $\operatorname{dom} C_{i} \subset \operatorname{dom} C_{i}^{*}$. Para eso basta observar que para $(x, w)^{T}=z \in \operatorname{dom} C_{i}$ vale $\left\langle z, C_{i} z\right\rangle=<$ $C_{i} z, z>=c_{i} x^{2}$. i.e. $C_{i} \subset C_{i}^{*}$, luego $z \in \operatorname{dom} C_{i}^{*}$ y $C_{i} \subset C_{i}$.

Sea

$$
\left(\begin{array}{l}
u \\
v
\end{array}\right):=C_{i}^{*}\left(\begin{array}{l}
x_{2} \\
w_{2}
\end{array}\right) \text { y }\left(\begin{array}{l}
x_{1} \\
w_{1}
\end{array}\right) \in \operatorname{dom} C_{i}
$$

luego

$$
<\left(\begin{array}{l}
u \\
v
\end{array}\right),\left(\begin{array}{c}
x_{1} \\
w_{1}
\end{array}\right)>=<\left(\begin{array}{c}
x_{2} \\
w_{2}
\end{array}\right), C_{i}\left(\begin{array}{c}
x_{1} \\
w_{1}
\end{array}\right)>
$$

i.e. $M u x_{1}+\rho \int_{0}^{L} v(z) w_{1}(z)=x_{2} c_{i} x_{1}$, que factorizando tenemos

$$
\left\{M u-c_{i} x_{2}\right\} x_{1}+\rho \int_{0}^{L} v(z) w_{1}(z) d z=0
$$


En particular, para $x_{1}=M u-c_{i} x_{2}$ e $w_{1}=v$ en (??) tenemos

$$
\left(M u-c_{i} x_{2}\right)^{2}+\rho \int_{0}^{L}(v(z))^{2} d z=0 .
$$

Luego, $u=c_{i} M^{-1} x_{2}$ y $v=0$, i.e. $(u, v)^{T}=C_{i}^{*}\left(\left(x_{2}, w_{2}\right)^{T}\right)=C_{i}\left(\left(x_{2}, w_{2}\right)^{T}\right)$, i.e. $C_{i}^{*} \subset C_{i}$.

Por lo tanto, $C_{i}^{*}=C_{i}$.

Proposición 3.3. Se verifican los siguientes resultados

i) $\operatorname{Ker} A=\left\{(x, w)^{T} \in \operatorname{dom} A \subset X, w(z)=x, \forall z\right\}$.

ii) $\operatorname{Ker} C_{0} \cap \operatorname{Ker} A=\{0\}$.

iii) El subespacio $\operatorname{Ker} C_{1} \cap \operatorname{Ker} A$ no contiene autovectores de $C_{0}$.

\section{Prueba.}

i) En efecto, sea $Z=(x, w)^{T} \in \operatorname{Ker} A$, entonces $A Z=0$, luego, $w^{\prime}(0)=0$ y $w^{\prime \prime}=0$, entonces $w^{\prime}(\xi)-w^{\prime}(0)=0$, i.e. $w^{\prime}(\xi)=w^{\prime}(0)=0$. Así, $w(\xi)=$ constante, pero $w(0)=x$, luego, $w(\xi)=x$.

ii) En efecto, sea $z=(x, w)^{T} \in \operatorname{Ker} C_{0} \cap \operatorname{Ker} A$ entonces $c_{0} M^{-1} x=0$, esto es $x=0$, por otro lado $A z=0$, luego $w(\xi)=0, \forall \xi \in[0, L]$, i.e. $w=0$.

iii) En efecto, supongamos que $Z=(x, w)^{T}$ sea un autovector de $C_{0}$, (i.e. $Z=(x, w)^{T} \neq 0$ y $C_{0} Z=\lambda Z$ ) tal que $Z \in \operatorname{Ker}_{1} \cap \operatorname{Ker} A$,

$$
\begin{gathered}
0=C_{1} Z=\left(M^{-1} c_{1} x, 0\right)^{T} \Rightarrow x=0, \\
0=A Z \Rightarrow w(\xi)=x=0, \forall \xi \text { i.e. } w=0,
\end{gathered}
$$

i.e. $Z=0$, lo que es absurdo.

Ahora presentamos el principal resultado del trabajo.

Teorema 3.1. El sistema (??), (??), (??) decae exponencialmente.

Prueba. El sistema (??), (??), (??), reescrito como (??), satisface las hipótesis del Teorema ??, luego el sistema decae exponencialmente.

Observación 3.2. Bajo un control adecuado, se consigue estabilizar exponencialmente la rotación de un satélite con apéndices flexibles (modelo extraido de Skaar [18]).

Su prueba es similar al del Teorema ??. 


\section{Bibliografía}

[1] Desh W. and Grimmer.(1987). Propagation of singularities for integrodifferential equation. J. Differential Equation.

[2] Desh W., Grimmer and Schappacher.(1984). Some considerations for linear integrodifferential equation. J. Math Anal. Appl. 104: 219- 234.

[3] Hrusa W. and Renardy M.(1985). On wave propagation in linear viscoelasticity. Quart. Appl. Math. 43: $237-254$.

[4] Miller R.(1975). Volterra integral equation in a Banach Space. Funkcial Ekvac 18: 163-194.

[5] Miller R. and Sell G.(1970). Volterra integral equations and topological dynamics. Mem. Amer. Math. Soc 102.

[6] Miller R. and Wheeler R.(1978). Wellposedness and stability of linear Volterra integrodifferential equations in abstract spaces, Functial Ekvar 21: 279 - 305.

[7] Nagel R.(1986). One parameter semigroups of positive Operatoprs. Lecture Notes in Math, 1184, Springer, Berlin/ New York.

[8] Pazy A.(1983). Semigroups of linear operator and applications to partial differential equations. Applied Mathematical Sciences. 44 Springer Verlag, Berlin.

[9] Prub J.(1983). Stability analysis for equilibria in age specific populations dynamics. Nonlinear Anal. T. M. A. 7.

[10] Russel D. L.(1978). Controllability and stabilizability theory for linear partial differential equations. Recent progress and open questions. SIAM Rev. 20: 639-739.

[11] Santiago Y.(2002). Global existence and exponential decay to the wave equation with localized frictional damping. PESQUIMAT Revista de la Fac. CC. MM. de la UNMSM. Vol V. Nro. 2: 1-19.

[12] Santiago Y.(2004). Estabilidad exponencial del Semigrupo $C_{0}$ asociado aun sistema Termoelástico. PESQUIMAT Revista de la Fac. CC. MM. de la UNMSM. Vol VII , Nro. 1: 30-42.

[13] Santiago Y.(2005). Decaimiento exponencial de la solución débil de una ecuación de la Onda no lineal. PESQUIMAT Revista de la Fac. CC. MM. de la UNMSM. Vol VIII, Nro. 2: 29 - 43.

[14] Santiago Y.(2007). About decay of solution of the wave equation with dissipation. PROYECCIONES Journal of Mathematics, Vol. 26, Nro. 1: 37-71.

[15] Santiago Y.(2009). Control en la frontera libre de una viga viscoelástica con memoria. PESQUIMAT Revista de la Fac. CC. MM. de la UNMSM. Vol XII Nro. 1: 32-49.

[16] Schappacher W.(1983). Asymptotic behavior of linear $C_{0}$ Semigroups. Quaderni Bari 1/83.

[17] Skaar S.(1984). Closed form optimal control solutions for continuous linear elastic systems. J. Aeronaut Sci 32: 447-461.

[18] Skaar S., Tang L. and Yalda-Mooshabad I.(1986). Observations regarding on off attitude control of flexible satellites, Preprint 86062, Engineering Research Institute, Iowa State University. 
[19] Werb G.(1985). Theory of nonlinear age- dependent population Dynamics. Monographs and Textbooks in Pure and Applied Mathematics Series 89, Dekker, New York. 